\title{
Vivienda social periurbana en Santiago de Chile: la exclusión a escala regional del trasurbanita de Santiago de Chile
}

\section{Peri-urban social housing in Santiago de Chile: the exclusion at regional scale of the trasurbanita of Santiago de Chile}

César Cáceres-Seguel*

\begin{abstract}
The location of peri-urban social housing in Santiago de Chile has been broadly studied from political and territorial implications, however, little do we know about the impact of urbanization in the quality of life of the inhabitants. By means of interviewing residents of projects located in Lampa, it is suggested the rise of a lowincome trasurbanitas. For them, access to a basic wellbeing depends on inhabiting daily fragmented territories between the peri-urban home and central communities of Santiago, where basic services are concentrated. The housing subsidy triggers a regional exclusion of groups inhabiting peri-urban edges lacking in efficient public transport and basic services.
\end{abstract}

Keywords: social housing, peri-urbanization, quality of life, Santiago de Chile, social exclusion.

\section{Resumen}

La localización periurbana de vivienda social en Santiago de Chile ha sido ampliamente estudiada desde sus implicancias territoriales y políticas, sin embargo, poco sabemos del impacto de estas urbanizaciones en la calidad de vida del habitante. Mediante entrevistas con residentes de proyectos localizados en Lampa se sugiere el surgimiento de trasurbanitas, para ellos, el acceso a un bienestar básico depende de habitar cotidianamente territorios fragmentados entre la vivienda periurbana y comunas centrales que albergan servicios urbanos elementales. El subsidio de vivienda desencadena la exclusión a escala regional con grupos habitando bordes periurbanos carentes de transporte público eficiente y servicios básicos.

Palabras clave: vivienda social, periurbanización, calidad de vida, Santiago de Chile, exclusión social.

\footnotetext{
* Centro de Inclusión e Innovación Social, Universidad Viña de Mar, Chile, correo-e: cesar.caceres@gmx.com
} 


\section{Introducción}

Históricamente, la comprensión y gestión de la ciudad se funda desde una dualidad urbano-rural. Sin embargo, la multiplicación de proyectos residenciales y de consumo, desarrollados en los bordes metropolitanos, no sostiene más esta noción de urbanidad y ruralidad como espacios aislados (Fishman, 1987; Garreau, 1991; Tacoli, 1998; Iaquinta y Drescher, 2000; Sieverts, 2003; Indovina, 1998, 2007 y Soja, 2011).

Los modos flexibles de localización de actividades económicas y población en el territorio definen al espacio periurbano como un territorio en transición sometido a intensivos procesos de transformación de suelo rural en áreas urbanas (Allen 2003: 136 y McGregor et al, 2012). Siguiendo a Indovina (1998: 24 y 2007: 14), la expansión de la ciudad en los bordes metropolitanos se configura desde los elementos propios de la constitución de la ciudad, aun cuando no muestran las características de densidad, intensidad y solución de continuidad típicos de éstas. El surgimiento de la ciudad periurbana está asociado en buena medida a las nuevas infraestructuras de movilidad física y virtual, que han sido capaces de transformar los principios de localización sobre el territorio de individuos y actividades; en consecuencia, la ciudad se ha vuelto un producto altamente maleable (Sieverts, 2003: 71).

La nueva relación individuo-espacio impulsa un proceso de urbanización regional que se distancia de la noción de metrópolis monocéntrica, se transita hacia un ciclo de ciudad-región compuesta por núcleos urbanos organizados en red (Soja, 2011: 684); un ciclo de expansión metropolitana que conlleva la aparición de un habitante que no necesariamente interactúa con sus vecinos, sino que establece prácticas de vida cotidiana flexibles y multi-escalares (Martinotti, 1993; Ascher, 2004 y Muńoz, 2008).

Para Martinotti (1993), esta nueva fisonomía urbana más extendida y multinuclear conlleva la aparición de nuevas tipologías de habitante urbano. Si la ciudad en sus primeras fases mostró un habitante que residía en la misma ciudad que trabajaba o consumía, la metrópolis de post guerra aparece unida a un commuter que trabaja en áreas centrales pero reside en la suburbia.

Hoy, la metrópolis se vuelve flexible, tanto en estructura como en el perfil de habitante, se agrega un tipo de "habitante-usuario" (Martinotti, 1993: 146) que no reside ni trabaja en la ciudad, pero que hace un uso intensivo de servicios y equipamiento localizados en ésta. Al profundizar en la descripción de este habitante metropolitano actual, Muñoz (2008: 34) plantea el concepto de territoriante, asociado a un "habitante a tiempo parcial, que utiliza el territorio de dsitinta forma en función del mo- 
mento del día o del día de la semana y que, gracias a las mejoras en los transportes y las telecomunicaciones, pueden desarrollar diferentes actividades en puntos diferentes del territorio de una forma cotidiana (...) por tanto se define como territoriante entre lugares y no como habitante de un lugar".

Lo que aquí se plantea es que esta nueva morfología social de la metrópolis resulta clave para explicar y entender procesos de exclusión social en metrópolis latinoamericanas. En un contexto de ciudades cada vez más extensas y fragmentadas, el acceso a servicios y oportunidades urbanas resulta un asunto central para las políticas urbanas y sociales (Kaztman, 2001; Dureau et al., 2002; Galster y Killen, 1995).

En Latinoamérica, esta problemática adopta especial trascendencia dada la tendencia hacia la localización periférica de grupos socioeconómicamente bajos localizados en conjuntos de vivienda social (Caldeira, 2000; Taschner y Bogus; 2001; Janoschka, 2002; Rodriguez y Arriagada, 2004; Ludeña, 2006 y UN-Habitat, 2009).

La localización periurbana de vivienda social activa dinámicas de exclusión posibles de describir como dos fuerzas de claro contenido espacial: dispersión y concentración:

1. Exclusión mediante dispersión de grupos vulnerables en los bordes metropolitanos, que implica para estos grupos el distanciamiento de oportunidades educativas, laborales o comerciales localizadas exclusivamente dentro la ciudad (Massey y Denton, 1993; Taschner y Bogus, 2001; Macgregor, 2012; Hanhörster, 2001 y Madanipour, 2011).

Bajo este enfoque, las oportunidades de vida de un individuo o familia no sólo dependen del mercado laboral, sistema educativo o políticas de estado de bienestar, sino de cómo el mercado e instituciones se despliegan sobre el territorio (Galster y Killen, 1995). Se transita así hacia una noción de pobreza de conexiones, donde ciertos grupos muestran limitado potencial de movilidad para ejercer influencia sobre su espacio y tiempo (Graham y Marvin, 2001).

2. Las dinámicas de exclusión surgen basadas en la concentración de grupos vulnerables en áreas (o barrios) específicas de la ciudad. Estas áreas resultan residenciales de alta densidad, marcadas por el déficit de servicios y equipamiento barrial. La escasa oferta de oportunidades de recreación, salud, cultura y deporte en estas áreas de vivienda social refuerza las condiciones de inequidad estructural dadas por las brechas educativas y de ingreso presente en estas sociedades. Así, el menos privilegiado deviene aún menos privilegiado debido al acceso diferenciado a oportunidades recreativas, comerciales y de servicios en sus barrios (Ellen y Turner, 2001; Lupton, 2003; Forrest, 2008; Galster, 2010 y Knox y Pinch, 2014). 
El caso de Santiago de Chile resulta interesante de analizar desde la perspectiva del acceso a oportunidades urbanas, ya que su expansión periurbana reciente se asocia al desarrollo de proyectos de vivienda social. ${ }^{1}$ Históricamente, los gobiernos chilenos han considerado la política de vivienda uno de los pilares fundamentales de la política social del país. Desde los años treinta hasta los sesenta, los programas de vivienda debieron dar respuesta a un proceso intensivo de migración campo-rural. En los noventa, los gobiernos democráticos abordaron el déficit de vivienda -heredado de la dictadura de Pinochet- mediante un plan de inversión en el que se construyeron 1,270,000 viviendas entre 1992 y 2002 (para un país de 13,348,401 habitantes en 1992).

Esta fuerte inversión implicó que en 2002 sólo 2\% de la población nacional habitara en asentamientos informales. Asimismo, las viviendas con déficit de infraestructura básica (luz, alcantarillado, agua potable) se redujeron desde 616,000, en 1990, a 319,000, en el 2002. No obstante estos logros, autores plantean que en el caso de Santiago, los programas de vivienda social han activado procesos de segregación espacial al concentrarse principalmente en las periferias de las ciudades chilenas (Ducci, 1997; Sabatini et al, 2006; Hidalgo et al., 2007 y Brain y Mora, 2016).

Datos analizados por Hidalgo et al. (2007) plantean que entre 1990 y 2002 fueron construidas alrededor de 19,704 unidades de vivienda social en comunas periurbanas de Santiago. Asimismo Tokman (2006: 518) plantea que durante el $2001,85 \%$ de los lotes comprados para construir proyectos de vivienda social se concentraron en tres comunas periurbanas: Lampa, Buin y Paine. Este patrón de expulsión de la vivienda social fue legitimado desde la política pública, cuando en 2003 se modificó el artículo núm. 55 de la Ley General de Urbanismo y Construcción, ${ }^{2}$ que permitía la construcción de vivienda bajo 1,000 UF ${ }^{3}$ (rango de vivienda social) fuera de los límites urbanos.

${ }^{1}$ Durante la década del cincuenta la población urbana predomina por primera vez ante la rural. Década en la que Santiago concentra un 35\% de la población nacional. El 2015, de acuerdo con datos del Banco Mundial 89\% de la población chilena vive en ciudades. Década en la que Santiago ha aumentado su participación concentrando cerca de $40 \%$ de la población nacional.

Para una mejor comprensión de este proceso cabe señalar que la Región metropolitana de Santiago está compuesta por 52 comunas. El sistema urbano dentro de esta región está dividido en dos sistemas de asentamientos. La metrópolis continúa compuesta por 37 comunas denominada Gran Santiago (6,089,500 habitantes en 774,512 hectáreas), y 15 comunas periurbanas que rodean este núcleo metropolitano consolidado (794,063 habitantes en 357,156 hectáreas (figura 1).

2 "Fuera de los límites urbanos establecidos en los Planes Reguladores no será permitido abrir calles, subdividir para formar poblaciones, ni levantar construcciones, salvo aquellas que fueren necesarias para la explotación agrícola del inmueble, o para las viviendas del propietario del mismo y sus trabajadores, o para la construcción de conjuntos de viviendas sociales o de viviendas de hasta un valor de 1.000 unidades de fomento que cuenten con los requisitos para obtener el subsidio del Estado". (Ley 19,859, artículo núm. 55. D.O. 31.01.2003).

${ }^{3}$ Cuarenta mil dólares, aproximadamente. 
Entre la lógica detrás de esta normativa pro-dispersión se cuentan las limitaciones presupuestarias que muestra el subsidio para adquirir terrenos localizados dentro del Santiago consolidado. De acuerdo a Trivelli (2011: 179), desde los noventa, resulta imposible adquirir terrenos por menos de $1 \mathrm{UF} / \mathrm{m}^{2}{ }^{4}$ cifra inalcanzable para el monto asignado a cada subsidio familiar. ${ }^{5}$ Este desajuste entre subsidio y precio de suelo es uno de los factores fundamentales que explica la concentración de vivienda social en comunas periurbanas de la Región Metropolitana de Santiago (figura 1). Un dato que confirma esta tendencia lo entregan Brain y Sabatini (2006) al determinar que la distancia desde proyectos de vivienda social hacia el centro de Santiago (Plaza Italia) aumentó desde 20,8 kilómetros, en 1994, a 28,1 kilómetros, el 2004.

\section{Figura 1}

\section{Región Metropolitana de Santiago}

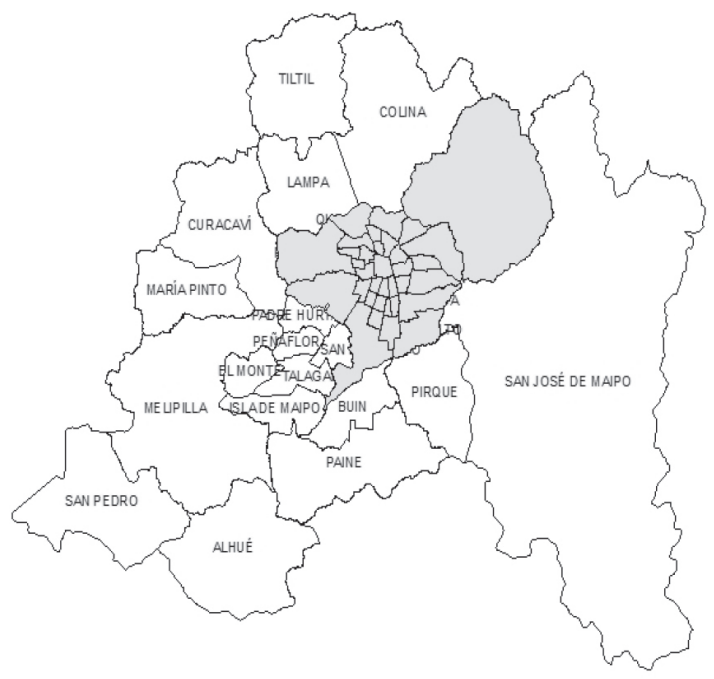

La Región Metropolitana de Santiago está compuesta por dos sistemas de asentamientos: una metrópolis consolidada denominada Gran Santiago (en gris) y comunas periurbanas (en blanco).

Fuente: elaboración propia.

Con el fin de investigar las condiciones de acceso a servicios urbanos básicos en urbanizaciones de vivienda social se estudiaron dos proyectos localizados en la comuna periurbana de Lampa. Lampa es una comuna de setenta y nueve mil habitantes aproximadamente, localizada a $37 \mathrm{ki}-$ lómetros al norte de la comuna de Santiago; representa un caso intere-

4 40,2 dólares, aproximadamente.

${ }^{5}$ Entre el 2002 y 2012, el precio de suelo dentro del Gran Santiago aumentó 44\% (Periódico La Tercera, 23 diciembre, 2012). 
sante de analizar dado que más de $60 \%$ de la producción de vivienda tiene menos de diez años.

Entre el 2002 y 2010 fueron construidas 2326 viviendas sociales que representan $18 \%$ de las viviendas construidas en dicho periodo en la Región Metropolitana de Santiago, cifra que se suma a las 1343 viviendas sociales construidas en el periodo 1980-2002 (Tapia, 2011: 116). Dado lo anterior, interesa analizar en este artículo de qué manera la localización de estas familias en Lampa implica una mejora de sus condiciones de vida o, más bien, representa una transformación de condiciones de precariedad.

Asociado a este proceso surgen preguntas como ¿qué prácticas cotidianas de acceso a atención de salud, educación o consumo caracterizan a este habitante?, ¿qué problemáticas sociales surgen de esta nueva vida en los márgenes periurbanos? y ¿qué lecciones se desprenden para la política urbana? Si bien el proceso de periurbanización en Santiago ha sido investigado desde la perspectiva de su proceso político y marco normativo (Salazar, 2010; Heinrichs et al., 2009 y Naranjo, 2008), así como desde el punto de vista de la localización de proyectos vivienda social (Tapia, 2011 e Hidalgo et al., 2007), resulta relevante comprender qué impacto tienen estas urbanizaciones en el acceso a servicios urbanos básicos y calidad de vida de grupos socioeconómicos bajos.

Los resultados de este artículo forman parte de la investigación doctoral "Exploring the periurban residential areas in Santiago de Chile: the asymmetric residential model between Corporate and State residential areas in Lampa 2000-2010", realizada por el autor en la Universität HCU Hamburg entre 2009 y 2013. La sección siguiente describe el marco analítico utilizado en la investigación; en la tercera sección se exhiben los principales resultados del proceso de entrevistas con habitantes. Finalmente se establecen las principales conclusiones y observaciones.

\section{Marco metodológico}

Este estudio parte de la noción de la metrópolis de Santiago como espacio social constituido por prácticas cotidianas llevadas a cabo por sujetos y colectivos. Para Lefebvre (2013: 97), la vida cotidiana del habitante surge de la vinculación del espacio percibido y la realidad material de lugares de trabajo y vida privada. Aquí interesa analizar aquellos lugares que permiten al habitante acceder a servicios urbanos básicos (salud, ocio, consumo, educación). Ahora bien, considerando que se trata de comunidades periurbanas, se define un modelo de análisis que reconoce el carácter multi-escalar de la vida cotidiana de estos grupos. Asimismo, este estudio se construye desde una noción de satisfacción residencial como un proceso evaluativo de las características sociales y materiales de la 
comunidad (Vidal y Pol, 2005: 283). La ciudad es producto de una dialéctica entre sociedad y espacio, por ende, se hace necesario estudiar la construcción de nuevas áreas residenciales desde el punto de vista de los actores involucrados en su producción.

Se evaluó para este fin el uso de entrevistas semiestructuradas como un método de análisis dentro de la geografía social (Kitchin y Tate, 2013), pertinente para investigar la vida urbana de estos grupos. Las entrevistas (30 personas en cada proyecto) se aplicaron en dos urbanizaciones de vivienda social: 1. Villa "El Pellín", construida durante la década del 2000, que cuenta con una población aproximada de 2100 habitantes provenientes de las comunas de Huechuraba, Lo Espejo, Macul, San Ramón, Lo Prado, Recoleta, así como familias provenientes de asentamientos irregulares (apropiación de terrenos) localizados en la comuna de Pudahuel y localizadas en el cerro San Cristobal (comuna de Santiago). 2. En el caso de la villa Isabel Riquelme, ésta alberga aproximadamente 3500 personas provenientes de las comunas de San Joaquín, Quinta Norma, Lo Espejo, Renca y Santiago (comunas internas del Gran Santiago).

Ambos proyectos se localizan dentro del centro urbano principal de la comuna de Lampa (figura 2). El cuestionario de preguntas abordó los siguientes temas: 1. El análisis de prácticas cotidianas de acceso a recreación, salud, educación y consumo, a escala local y metropolitana. 2. Aspectos subjetivos como percepción de seguridad, apego al barrio, y relación con vecinos. Para abordar estos asuntos se usaron dos tipos de preguntas: abiertas, donde el entrevistado evaluó asuntos subjetivos asociados con seguridad, satisfacción con el entorno residencial y calidad de vida, así como preguntas que incorporaron una escala de valoración que permitió tener una noción sobre la frecuencia de uso de espacios públicos, evaluación del equipamiento barrial y rutinas de acceso a atención de salud o centros de consumo (se adjunta el cuestionario de preguntas en el anexo 1. Las entrevistas se desarrollaron de septiembre a octubre del 2009 y de marzo a abril del 2010; tuvieron una duración de 30 a 40 minutos. El rango etario de los participantes se ubicó de los 25 a 60 años, aproximadamente. Las entrevistas se realizaron durante septiembre del 2010 y una segunda fase en marzo del 2011.

\section{Un subsidio sin llaves para la ciudad}

Para entender el proceso de relocalización de estas familias en Lampa cabe una breve referencia al proceso de obtención del subsidio habitacional. La postulación al denominado Fondo Solidario de Vivienda requirió, por parte de estas familias, un ahorro mínimo de 270 dólares aproximada- 


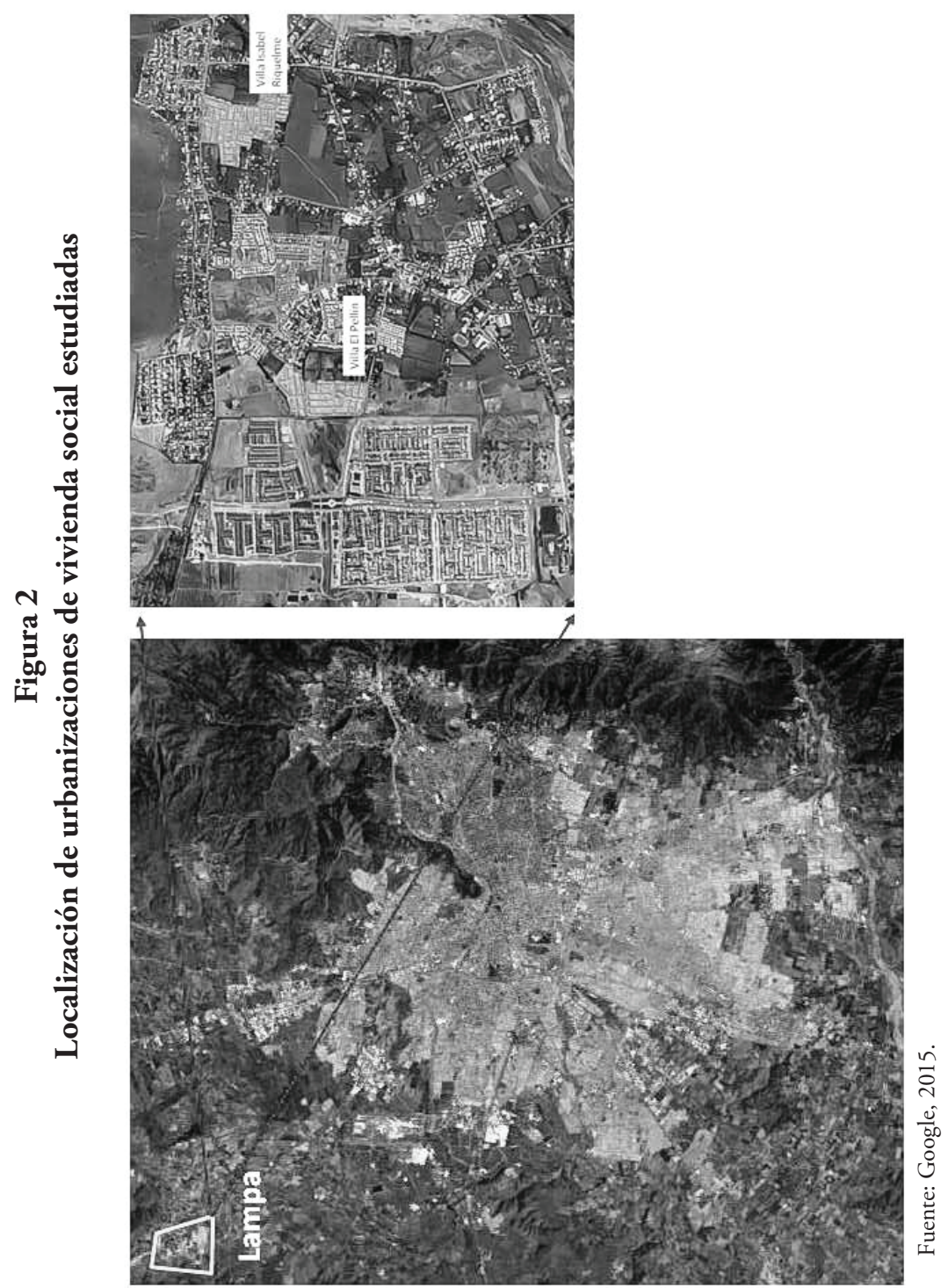


mente, así como su incorporación en un comité de vivienda formado por familias postulantes a la vivienda social.

$\mathrm{Al}$ momento de acceder al subsidio, la institución patrocinadora (municipio o servicio de vivienda y urbanismo) externaliza en una empresa constructora la tarea de encontrar un terreno y urbanizar el proyecto. Sobre el proceso de adquisición del terreno a urbanizar, los entrevistados plantean que la empresa comunicó las limitaciones presupuestarias de adquirir terrenos ubicados en comunas dentro del Gran Santiago. Se enfatizó que de acuerdo al monto de cada subsidio, las opciones de compra de terreno estaban restringidas a comunas periurbanas de la Región Metropolitana de Santiago, tales como Buin, San Bernardo, Lampa, Melipilla y Talagante (Figuras 3 y 4).

Llegué hace siete años desde Pedro Aguirre Cerda. Nos dijeron primero que el subsidio era para Buin, después nos dijeron que las casas estaban listas para Melipilla y después nos dijeron que era en Lampa, no había posibilidad de quedarme en Pedro Aguirre Cerda (Angela, 7 de abril de 2010, Villa Isabel Riquelme).

Llegamos hace un año y medio desde San Joaquín. Yo postulé al subsidio de casa usada, podría haber comprado en cualquier parte de la región metropolitana, pero el problema es que mi subsidio llegaba máximo a 9,600,000, y eso ya te deja pocas posibilidades, era la periferia sí o sí. Así que entre irme a Puente Alto que está peor catalogado preferí el norte. No había muchas más opciones por el precio, donde nosotros vivíamos las casas están en treinta millones, de ahí para arriba (Alejandra, 16 de septiembre de 2009, Villa Isabel Riquelme).

Figuras 3 y 4

\section{Villa El Pellín (izquierda) y Villa Isabel Riquelme (derecha)}
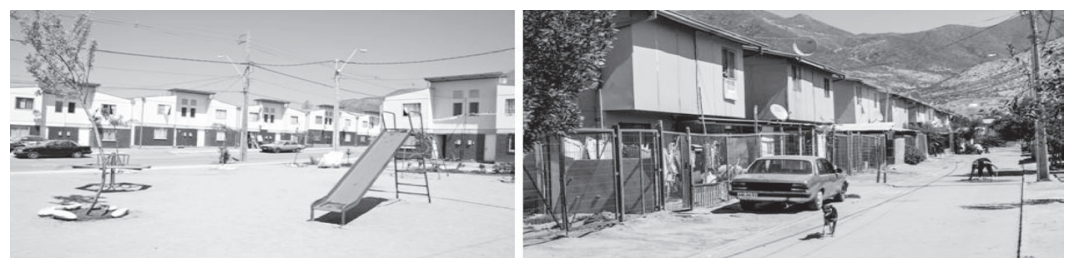

Fotografía tomada por el autor.

Dado lo anterior, se manifiestan las limitaciones presupuestarias que el subsidio familiar muestra para adquirir suelo localizado en comunas dentro del Gran Santiago. El precio del suelo en esta metrópolis actúa como una muralla invisible impidiendo el acceso de familias de estratos socioeconómicos más bajos a una vivienda localizada cercana a hospitales, empleo y variedad de comercio. Un primer punto a subrayar en el acceso 
a subsidio habitacional es que resulta un proceso donde las familias tienen nula capacidad de influir en la localización final de la vivienda.

\section{3. ¿Acceso a la vivienda o acceso al barrio?}

$\mathrm{Al}$ indagar en las prácticas recreacionales que el habitante despliega en su sector, emerge una experiencia residencial altamente condicionada por la calidad del equipamiento barrial presente. El acceso al subsidio habitacional se representó como la oportunidad de conquistar mejor nivel de equipamiento de barrio, sin embargo, esta expectativa se cumple parcialmente dado el deterioro de equipamiento recreacional como multicancha y áreas verdes. La inconformidad con el diseño del espacio público del proyecto se refleja en los siguientes reportes (figuras 5, 6 y 7):

Mira las multicanchas, ya no tienen bancas, no hay columpios, no tienen árboles, la municipalidad no las cuida. Hacia dentro del sector no hay ninguna plaza, es como un desierto [...] Si se mejoran los espacios públicos le cambiaría la mentalidad a la gente, te daría iniciativa de cuidarlo 'está bonita hay que mantenerlo'... pero así... qué ganas te dan de mantenerlo (Evelyn, 6 de septiembre de 2009, Villa Isabel Riquelme).

Las plazas no tienen algo bueno para uno ir y decir me voy a ir a sentar a la plaza. No me gusta caminar por mi barrio porque cuando yo quiero caminar pienso en un lugar agradable donde haya sombra en el camino, cosas para distraerse, pero aquí uno que ve casas y más casas (Irene, 16 de abril de 2010, Villa Isabel Riquelme).

Los resultados al explorar en rutinas de recreación son que 58\% de los entrevistados considera que los lugares recreativos están en mal estado, condición que explica que $74 \%$ señale no usar nunca dichos espacios. A diferencia de las familias de estrato medio o alto que acceden a valores residenciales superiores en proyectos periurbanos privados (Cáceres, 2015).

Las urbanizaciones de vivienda social estudiadas han sido diseñadas como extensos loteos basados en la repetición de viviendas semi-pareadas combinadas con pequeñas plazas de juegos infantiles. Si la literatura sobre espacios residenciales enfatiza el rol del equipamiento barrial en la satisfacción de necesidades cotidianas, la propuesta urbana detrás de estas urbanizaciones dirigidas por el Estado aparece desde un trazo urbano definible como una "ciudad desmantelada" de equipamiento barrial mínimo, por tanto incapaz de satisfacer necesidades humanas básicas. 


\section{Figura 5}

Plaza de juegos en villa Isabel Riquelme

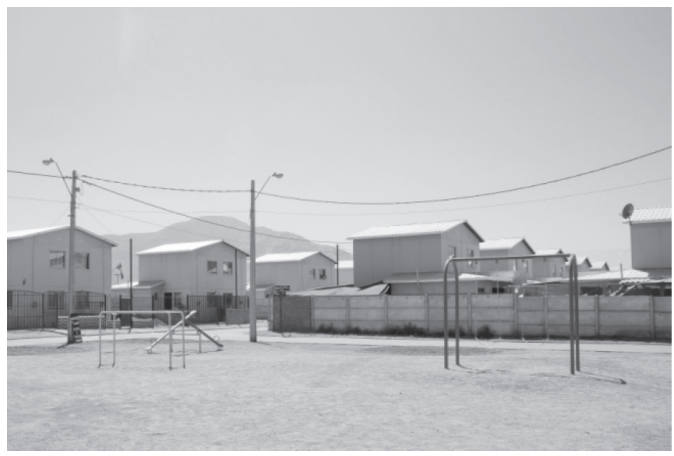

Fotografía tomada por el autor.

Figura 6

Plaza de juegos en villa El Pellín

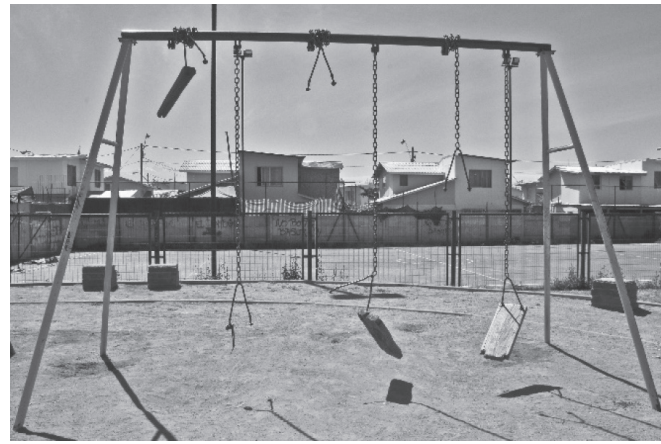

Fotografía tomada por el autor.

Figura 7

Nuevo proyecto de vivienda social en Lampa

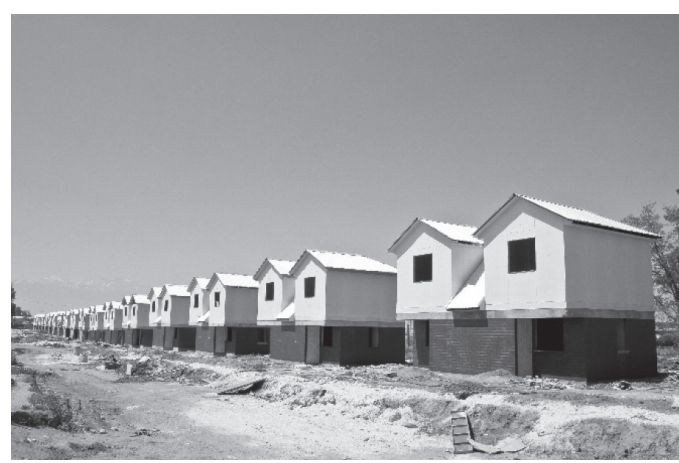

Fotografía tomada por el autor. 
Lo que surge del reporte del habitante es una una vida bajo régimen de urbanidad estatal que subvalora el rol social del entorno próximo. Esta ciudad desmantelada afecta especialmente a personas con un bajo potencial de movilidad (jóvenes madres, desempleados, niños, hombres y mujeres de tercera edad), personas para las que la oferta y calidad del equipamiento cercano resulta clave para la calidad de su experiencia cotidiana en la ciudad.

Para comprender la baja valorización de los espacios públicos caben los antecedentes que Brain y Sabatini (2006: 18) entregan con respecto al subsidio de vivienda. De acuerdo con los investigadores, del total del subsidio entregado a cada familia durante el 2004 (fecha aproximada de urbanización de estos proyectos), $20 \%$ del monto se utiliza para adquirir el suelo, 64\% para construcción de la casa, 13\% financia la urbanización básica (agua, gas, alcantarillado), y 1,9\% corresponde a inversión en equipamiento de barrio.

Esta baja inversión en equipamiento barrial entrega la responsabilidad de inversión a municipios, sin embargo, bajo el actual sistema de impuestos, viviendas de valor menor a 26,000 dólares aproximadamente están exentas del pago de impuesto territorial, hecho que daña la capacidad recaudatoria de municipios con alto nivel de vivienda social.

Lo problemático es que este bajo presupuesto asociado a equipamiento barrial no es corregido vía inversión ministerial o inversión directa vía planes estratégicos. Hoy, la política de desarrollo de barrios en Chile es una suma de programas dispersos e inconexos entre diversos ministerios y fondos concursables (educación, medio ambiente, cultura, deporte, salud, etc.). Predomina un modelo de habitabilidad vía voucher donde el desarrollo de barrios bien equipados depende de la capacidad de los gobiernos locales para adjudicarse fondos concursables administrados desde el gobierno regional. Esta problemática es identificada por funcionarios municipales en la comuna de estudio:

Hay un programa grande que se llama Programa de Mejoramiento Urbano con el que puedes hacer centros de internet, mejorar plazas, poner máquinas de ejercicios, áreas verdes, pero requiere que tengas un banco de proyectos, necesitas personas estudiando un proyecto de ciclovía, y eso no está. Además, los municipios compiten porque hay un presupuesto anual que da el gobierno donde todas las comunas postulan (Funcionario municipal, 16 de septiembre de 2009, Lampa).

En directa relación con el poco uso y valorización de los espacios públicos aparece en las entrevistas una percepción de inseguridad asociado al uso de estos lugares. El hecho de que 68\% de los entrevistados manifiesta sentirse inseguro en calles y espacios públicos conlleva una 
pérdida de significación como lugares de recreación y contacto entre vecinos. Asimismo, estimula el uso de dispositivos de seguridad en casas (rejas en ventanas) y cierre de pasajes, que acrecientan una condición de acuartelamiento en sus propios barrios. Aunque esta investigación no indaga en la real profundidad del problema de la delincuencia o drogadicción en estos lugares, la percepción de inseguridad por parte del habitante parece condicionar el contacto entre vecinos en espacios públicos.

Entraban muchos niños de afuera a fumar marihuana e hicieron tira las sedes, por eso nos pusimos de acuerdo para poner un portón que a las 10:00 pm se cierra. Desde ese momento está mejor (Ximena, 15 de septiembre de 2009, Villa El Pellín).

Tanto por la calidad del espacio público como por la percepción de inseguridad, estas urbanizaciones aparecen representadas como territorios deficitarios de lugares comunitarios significativos. La concepción del barrio como aquel lugar íntimo en lo extenso de la ciudad, que simboliza historias personales y vínculos sociales, parece no cumplirse en estas urbanizaciones. Desde el diseño urbano hasta la condición social integran un hábitat con débil capacidad de generar arraigo o sentido de pertenencia.

En la evaluación que el habitante hace de su entorno, surgen elementos de una cultura de gueto de refuerzo colectivo del desencanto con su lugar de vida. Reinterpretando a Auge (1993) y su definición de no lugar como aquel espacio no relacional, ni histórico ni identitario, cabe interpretar la vida en la vivienda social periurbana como una experiencia residencial en un no lugar incapaz de satisfacer necesidades cotidianas y promover arraigo. Si la anterior condición de allegado, o residente de "tomas" (slums) dañaba su vida familiar, su actual condición de habitante de macro extensiones de viviendas pareadas carentes de atributos de barrio, mella la construcción social de comunidad.

\section{Propietarios a 30 kilómetros de oportunidades sociales}

En la evaluación de su calidad de vida, el habitante manifiesta que la obtención de la vivienda propia trajo aparejada la redefinición de su vida en los bordes periurbanos de Santiago. Por ende, el acceso a hospital, comercio, educación y empleo se convirtió en factor determinante para su bienestar. Consultados sobre sus prácticas cotidianas de acceso a estos servicios, 91\% de los entrevistados ( $\Sigma 60)$ manifiesta que sus hijos (o nietos) accede a educación primaria o secundaria en la ciudad de Lampa, mientras que $9 \%$ lo hace en comunas localizadas dentro del Gran Santiago. 
En el caso de la atención a la salud, $97 \%$ accede a ella en hospitales públicos localizados en comunas céntricas del Gran Santiago como Recoleta (Hospital Salvador, Roberto del Río), mientras que 3\% acude a centros privados también localizados en comunas del Gran Santiago. Este punto es subrayado por los entrevistados como un factor de grave deterioro de su calidad de vida, ya que a nivel local sólo disponen de una posta rural sin especialistas médicos. La inexistencia de un centro de salud de mayor complejidad obliga a estos grupos a una movilidad intensiva, así como enfrentar situaciones de emergencia sin centros especializados a menos de 30 kilómetros.

[Atención médica] Si necesitas un tratamiento especial te mandan al San José (hospital público). Yo voy todas las semanas a Santiago porque los doctores nos dicen que tenemos que volver y uno tiene que ir [...] Yo volvería a Santiago porque todos los médicos están allá, acá uno gasta la plata viajando. Viajo como mínimo tres días a la semana por razonas de salud o compras. Debo tener 2,000 pesos para cada viaje, eso tres veces a la semana es caro, son como 25,000 pesos en transporte al mes, es mucho para mi sueldo (Beatriz, 31 de marzo de 2010, Villa El Pellín).

[Atención médica] La salud es mala en Lampa porque hay pocos especialistas. Por eso voy al San José (hospital) en Santiago [...] La desventaja de vivir en Lampa es que todo debes hacerlo en Santiago, el problema es que después de las 9:00 de la noche no puedes moverte de aquí porque no hay buses (Silvia, $6 \mathrm{de}$ abril de 2010, Villa Isabel Riquelme).

En el caso del acceso a productos básicos, 64\% de los habitantes entrevistados realiza compras en supermercados mayoristas y ferias específicas como La Vega, ambos localizados en las céntricas comunas de Recoleta y Santiago. Mientras que 36\% accede a productos básicos en comercio localizado en Lampa. El reporte muestra que aun existiendo supermercados en Lampa, el habitante prefiere desplazarse a comunas centrales de Santiago porque allí encuentra precios bajos (figura 8).

Yo voy dos veces a la semana, por el médico y cuando voy a hacer las compras [compra de productos básicos] [...] Lampa es muy caro, por eso voy a comprar mercadería a Santiago. La gente prefiere ir a La Vega porque sale más barato, compras más y traes más cosas. [Atención médica] Mandan al Roberto del Río o al San José, porque aquí en Lampa es sólo consultorio. No hay para hacerse radiografías y esas cosas, hay que ir siempre a Santiago (Gabriela, 5 de abril de 2010, Villa El Pellín).

[Compra de productos básicos] Yo no compro en el UNIMARC (supermercado) de Lampa porque es caro, por eso cuando voy los sábados a Santiago aprovecho de comprar y traer cosas. Incluso la feria es cara en Lampa, comparo el precio de las verduras con Santiago y es más caro. [Acceso a educación] Mis hijos viajan todos los días a Santiago porque uno estudia en el liceo y otro trabaja [...] En 


\section{Figura 8}

\section{Geografía de oportunidades y rutinas de acceso a servicios por parte de habitantes de proyectos de vivienda social en Lampa}

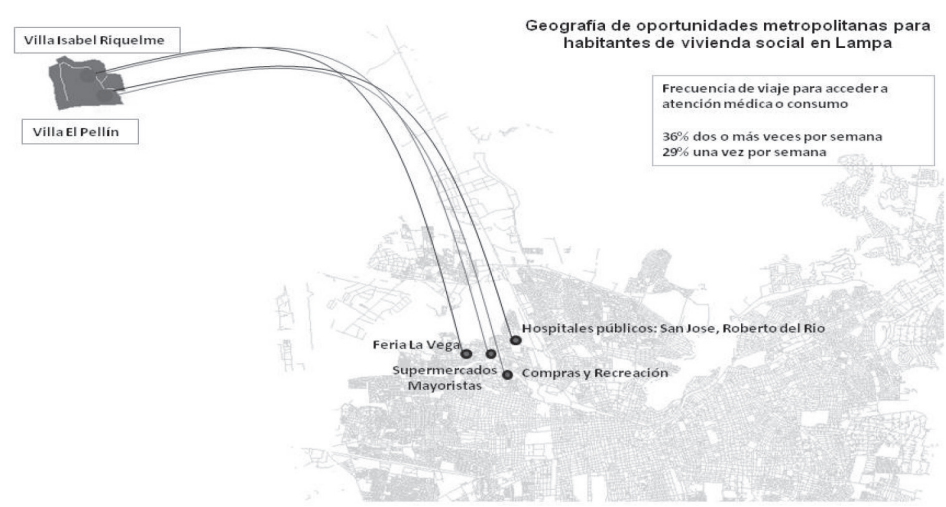

Fuente: Google, 2015.

Lampa yo perdí las comodidades, la locomoción, el comercio y lo barato de Santiago (Ángela, 15 de octubre de 2009, Villa Isabel Riquelme).

Esta vida altamente dependiente de comunas centrales del Gran Santiago deviene en una precaria existencia al considerar que Lampa resulta una comuna excluida de la cobertura del servicio de transporte público metropolitano denominado Transantiago (figura 9). Para desplazarse a Santiago estos habitantes disponen de un servicio de buses privado que conlleva un doble pago por trayecto (bus Lampa + Transantiago), así como la inexistencia de servicio de buses nocturnos.

De acuerdo al reporte del habitante, la inexistencia de un Transantiago tiene al menos tres efectos: 1 . Ante situaciones de emergencias médicas nocturnas, éstas familias deben recurrir al pago de automóviles particulares o de servicio de taxis; 2. Impacta en el acceso a oportunidades laborales y de estudios vespertinos; 3. El doble pago de pasaje resulta un costo demasiado alto para personas que fundamentalmente acceden a los niveles más bajos de remuneración en el mercado laboral de Santiago. En las entrevistas queda de manifiesto este conflicto:

[Calidad de transporte público] Es caro y malo, va a llegar a 1,000 pesos por viaje, ellos no piensan en la gente que viaja todos los días, mi esposo gasta 70,000 pesos y gana como 200,000 mensual más o menos [...] Mi hijo ganó una beca para estudiar inglés en Santiago, pero no pudo tomarla porque el último bus se viene como a las 23:30. Si estudiaba no alcanzaría el bus para llegar a la casa, por ese motivo mi hijo tuvo que rechazar la beca (Irene, 5 de abril de 2010, Villa Isabel Riquelme). 


\section{Figura 9}

\section{Desajuste espacial entre la localización de vivienda periurbana y cobertura del sistema de transporte metropolitano TRANSANTIAGO}

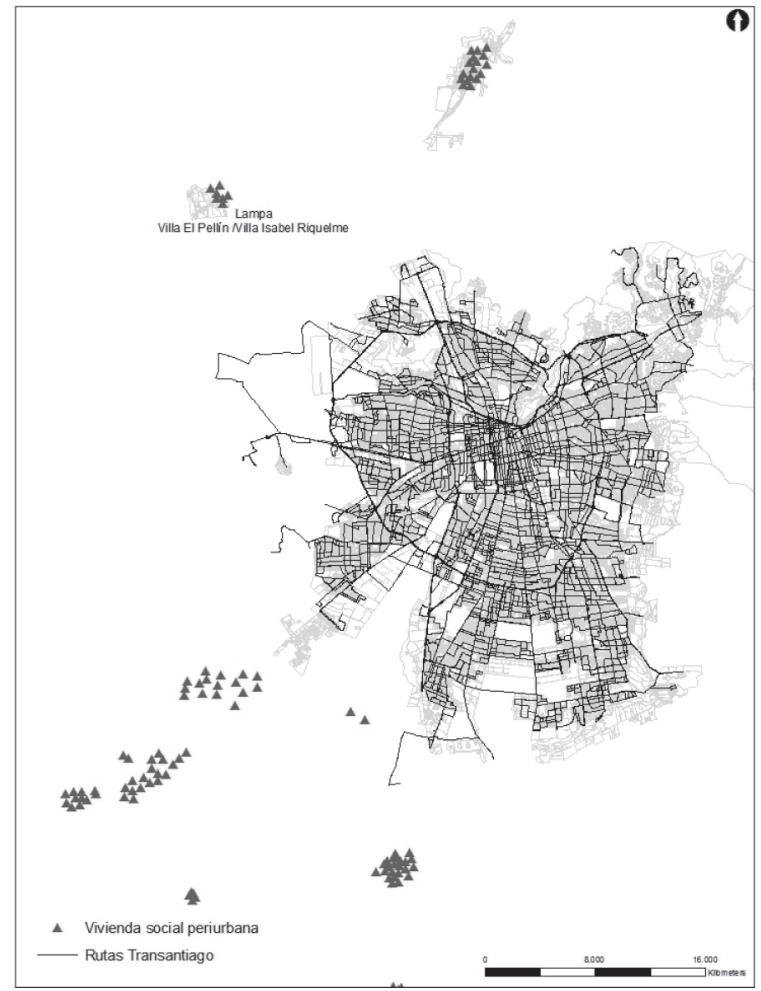

Fuente: elaboración del autor a partir de cartografía facilitada por el laboratorio urbano del Instituto de Geografía de la Universidad Católica de Chile.

[Calidad de transporte público] Yo estuve como seis meses trabajando en Santiago, trabajaba en Lo Espejo (Comuna), gastaba 4,000 pesos diarios en transporte y ganaba el mínimo, sacaba 150,000. Gastaba 80,000 al mes en transporte (Rubén, 16 de abril de 2010, Villa El Pellín).

Yo llegue en el 2004 y hasta el 2008 viaje a Santiago. Me tenía que levantar a las cinco de la mañana y llegaba a las nueve de la noche, aunque de mi trabajo salía a las tres. No es vida estar cinco horas arriba de una micro, o esperar una hora y medio a la micro para poder llegar (Gloria, 8 de abril de 2010, Villa El Pellín).

¿Cómo definir esta nueva categoría de habitante del Gran Santiago? La localización periurbana de la vivienda social trae consigo el surgimiento de un tipo de habitante que para acceder a un bienestar mínimo debe 
adoptar una vida a escala regional, residiendo en el hogar periurbano y convirtiéndose al mismo tiempo en habitante diurno, flotante pero intensivo de servicios localizados en comunas centrales del Gran Santiago, por ejemplo, hospitales públicos, ferias, supermercados mayoristas y retail, etc. Para estas familias, las comunas céntricas del Gran Santiago (Recoleta y Santiago) se representan como centros de oportunidades comerciales y de servicios urbanos. Por ello dibujan cotidianamente cartografías bidireccionales y estandarizadas de acceso a esta urbanidad básica (figura 8).

Mi vida aquí mejoró en que ya no soy allegada y la calidad del aire es distinta, pero perdí el acceso a los mayoristas que tenía muy cerca de la casa que tenían buenos precios, también perdí el hospital que me correspondía, todas esas cosas las trato de resolver igual en Santiago (Alejandra, 7 de abril de 2010, Villa Isabel Riquelme).

A mí me ha costado porque tengo mi familia en Santiago, incluso cuando llegué aquí me dio depresión [...] no me proyecto acá porque me ha costado salir adelante, yo en Santiago tenía mi trabajo, trabajé de guardia, de supervisora en una empresa de aseo, entonces nos vinimos para acá y todo cambió. Hay oferta de trabajo en Santiago pero es difícil porque casi siempre ofrecen el mínimo, entonces uno ya tiene que descontar treinta y tantos mil de ese sueldo mínimo, hay que buscarse algo por acá cerca. Si me dieran la posibilidad si volvería. Mi vida aquí mejoró porque tengo mi casa pero la parte económica fue un desastre (María, 6 de octubre de 2009, Villa El Pellín).

He perdido porque me vine muy lejos, tengo que estar gastando mucha plata para salir a Santiago. A mi familia la veo muy a lo lejos porque es más plata, son como 1,500 pesos cada pasaje y nosotros somos cinco. Acá hay trabajo sólo en la temporada de verano, en el invierno la gente queda cesante, eso no piensa el gobierno (Fabiola, 29 de marzo de 2010, Villa El Pellín).

La vivienda propia significó la superación de una precariedad extrema, sin embargo, distanció a estos grupos de los lugares más significativos para su subsistencia: cercanía a familiares, feria, supermercado mayorista. Dejar atrás la precariedad extrema de asentamientos informales o de allegados significó para estas familias convertirse en trashumantes en su propia ciudad.

La expansión periurbana de la vivienda social redefine la morfología social de Santiago sumando una categoría de trasurbanita que para acceder a una urbanidad elemental debe habitar cotidianamente una ciudad hecha de territorios fragmentados y distantes entre sí. La adjudicación de la vivienda propia luego de unos ańos se representa como un capital que les permite superar la ciudad informal pero los excluye de una urbanidad básica, condenándolos a una condición de trasurbanitas administrando una vida in between, solo administrable mediante una intensiva movilidad 
diaria. Este distanciamiento del sistema de oportunidades denominado Gran Santiago explica porque 64\% de los entrevistados desea abandonar su vivienda y regresar al Gran Santiago.

Yo era del campamento más grande de Chile, en Pudahuel [...] cuando llegué aquí me dio depresión, no le tenía amor a mi casa, lloraba todos los días. Nunca me gustó acá, siempre me venía llorando de mi trabajo. A todo el campamento deberían habernos dado casa en una parte y no habernos desparramado [...] Si pudiera volvería, aquí no me gusta, si puedo más adelante venderé mi casa y me iré porque aquí no hay futuro, aquí no hay empleo, aquí la gente la mayoría son temporeros (Silvana, 12 de abril de 2010, Villa Isabel Riquelme).

\begin{abstract}
Vengo del campamento senador mate en Recoleta [...] si pudiese volvería a Santiago porque toda mi familia está allá, además perdí las comodidades que uno tenía allá, en Santiago había más locomoción para salir, yo estaba más cerca del centro, acá no [...] pero es difícil porque yo soy propietaria acá, tendría que vender, pero acá las casas nadie las va a comprar (Aída, 16 de septiembre, de 2009, Villa El Pellin).
\end{abstract}

Estas familias no buscaron convertirse en periurbanitas porque precisamente de la cercanía, o fácil accesibilidad, dependen sus redes de supervivencia. El habitante de proyectos periurbanos privados si decide regresar al Gran Santiago vende la vivienda y regresa, sin embargo, para el periurbanita de vivienda social la baja valorización de su vivienda en el mercado les impide regresar al Gran Santiago. Son prisioneros de un subsidio que los condena a una pobreza de conexiones a elementos elementales de urbanidad, una pobreza disfrazada por su condición de propietarios de vivienda social.

Tradicionalmente existe en el imaginario del habitante de Santiago la noción de dos ciudades conviviendo en una misma urbe, se habla "de plaza Italia para arriba” para señalar donde residen las clases medias altas y altas; así como se acuñó el término "de plaza Italia para abajo" para referirse al lugar de residencia de los grupos más vulnerables. Pues bien, aunque esta geografía social aún persiste no da cuenta cabal de las nuevas cartografías de exclusión en el Santiago metropolitano.

Los programas de vivienda social localizados han transformado la morfología social de la metrópolis instalando grupos vulnerables en áreas periurbanas deficitarias de servicios básicos y empleo. La política de vivienda social en su lucha contra la pobreza ha generado nuevas formas de exclusión de grupos de estrato bajo carentes de elementos básicos de ciudad. 


\section{Conclusiones}

El problema social que conlleva el surgimiento de este trasurbanita, de estrato socioeconómico bajo, que habita la vivienda social periurbana implica al menos tres grandes desafíos para la política urbana del Santiago metropolitano.

1. Derribar la muralla invisible dada por el precio de suelo. Las condiciones de vida de habitantes de vivienda social en Lampa entregan evidencia empírica que refuerza la necesidad de arribar a nuevos instrumentos técnicos-financieros que permitan una localización de proyectos de vivienda social en comunas situadas dentro del Gran Santiago, esto es: propuestas como que los nuevos desarrollos inmobiliarios cedan 5\% de la superficie total del proyecto para la construcción de vivienda social y la generación de zonas de interés social con porcentajes de viviendas de interés público dentro de comunas del Gran Santiago.

En el caso del subsidio a la localización -creado en 2006 para facilitar la construcción de proyectos en terrenos bien localizados- no muestra un real impacto en metrópolis como Santiago, dado que sus indicaciones de estar a 500 metros de transporte público o 2500 metros de un centro de salud primario, resultan insuficientes si consideramos que dentro de estos parámetros nuevos proyectos en Lampa se considerarían dentro de criterios aceptables.

Considerando lo expuesto en este estudio, 500 metros de paradero de Transantiago sería probablemente un parámetro adecuado para asegurar una correcta localización de este tipo de proyecto. Asimismo, como se destaca en la propuesta de política de suelo (Propuesta de política de suelo, MINVU 2015), Chile no cuenta con mecanismos regulatorios eficientes, sean de tipo tributario, de planificación urbana, o gestión del suelo, capaces de proveer de suelo para responder a la demanda futura y prevenir tendencias de segregación social.

No obstante las anteriores propuestas, la política urbana debe hacerse cargo de las miles de familias ya localizadas en comunas periurbanas, personas que difícilmente obtendrán un nuevo subsidio.

2. Transantiago periurbano y el derecho a la movilidad. El origen de la precariedad mostrada por el trasurbanita de vivienda social se asocia tanto a la localización periurbana de su vivienda como a la no incorporación de Lampa (y otras comunas periurbanas: Colina, Padre Hurtado, etc.) a la cobertura del sistema metropolitano de transporte Transantiago. Esta descoordinación entre política de vivienda y transporte implica para familias vulnerables el doble pago por tramo. Un monto importante considerando sus ingresos mensuales. Si atendemos a los costos del transporte periurbano, y ausencia de servicio nocturno, resulta urgente activar 
la discusión sobre la extensión del Transantiago a comunas periurbanas como Lampa.

3. Hacia una concentración descentralizada de atributos urbanos. La insostenibilidad del modelo de expansión residencial de Santiago no radica únicamente en la expulsión de los pobres hacia los bordes periurbanos, sino también en no descentralizar servicios básicos mediante nuevos centros periurbanos. La expansión natural de la metrópolis y precio de suelo hacen necesario pensar la metrópolis como un sistema de centralidades comunales y barriales jerárquicas e interconectadas. Aunque actualmente Santiago tiende a la compactación y crecimiento de comunas centrales, no es menos cierto que la expansión de proyectos residenciales en comunas periurbanas transforma la geografía de necesidades sociales en la metrópolis. El ciclo de urbanización periurbana, aunque menos masivo y elocuente que la urbanización dentro del Gran Santiago, será un ámbito de acción estratégico en la agenda política urbana de Santiago las próximas décadas. Como advierte el surgimiento del trasurbanita de vivienda social periurbana, reproducir calidad urbana a los bordes periurbanos de Santiago será tan relevante en los próximos ańos como producirla en comunas centrales densamente pobladas.

\section{Anexo I}

\section{Cuestionario de Preguntas}

1. For how long do you live in this project?

2. Where did you live before?

3. Did you apply to Lampa or was this your only option?

4. Do you or a family member work in Santiago?

5. Is a member of your family in a school or a kindergarten of Lampa?

6. How do you evaluate the schools or kindergarten of Lampa?

7. When you receive medical attention where do you go most frequently? Is it a private or a public institution?

8. About the medical center: How often do you attend medical centers?

9. Have you attended the public health post of Lampa? If not, why?

10. Where do you shop your groceries?

11. How often do you shop in a supermarket?

12. How often do you use the supermarket of Lampa? 
13. How do you evaluate the commercial offer in Lampa?

14. How do you evaluate the quality of squares and multipurpose courts in your neighborhood? Very good - good - medium - poor - very poor?

15. How often do you use squares and parks of your neighborhood? Every day - more than three times a week - at weekends - twice per month?

16. What recreational activities do you make in your neighborhood?

17. Why are the squares destroyed? Give your opinion?

18. Which means of transport do you use to go to Santiago?

19. How often do you go to Santiago for different reasons than the job? Which needs produce the highest demand for mobility?

20. Name the facilities that are necessary to improve your neighborhood today?

21. Do you participate in a neighborhood board?

22. How would you describe your relationship to your neighbors?

23. If you see problems with the quality of squares, holes in the streets, lighting, etc., do you contact the municipality?

24. Do you project your future in this neighborhood?

25. Do you feel attached to this neighborhood?

26. Do you feel safe living here? Do you feel safe walking at night?

27. Did your life quality improve or worsen when you came to this project? What are the main differences? 


\begin{tabular}{lc}
\hline \multicolumn{1}{c}{ Nombre } & Villa El Pellin \\
Ingrid & Villa \\
Teresa & Villa El Pellin \\
Gabriela & Villa El Pellin \\
Magdalena & Villa El Pellin \\
María & Villa El Pellin \\
Gloria & Villa El Pellin \\
Aída & Villa El Pellin \\
Evelyn & Villa El Pellin \\
Marion & Villa El Pellin \\
Beatriz & Villa El Pellin \\
Sandra & Villa El Pellin \\
Fabiola & Villa El Pellin \\
Rubén & Villa El Pellin \\
Sonia & Villa El Pellin \\
Margot & Villa El Pellin \\
Gladys & Villa El Pellin \\
Rocío & Villa El Pellin \\
Inés & Villa El Pellin \\
Macarena & Villa El Pellin \\
Silvana & Villa El Pellin \\
Melisa & Villa El Pellin \\
Cintia & Villa El Pellin \\
Debora & Villa El Pellin \\
Patricia & Villa El Pellin \\
Fernando & Villa El Pellin \\
Carlos & Villa El Pellin \\
Mariela & Villa El Pellin \\
Ximena & Villa El Pellin \\
Eduardo & Villa El Pellin \\
Mónica & Villa El Pellin \\
\hline & Villa El Pellin \\
\hline
\end{tabular}

\begin{tabular}{lc}
\hline \multicolumn{1}{c}{ Isabel Riquelme } \\
\hline \multicolumn{1}{c}{ Nombre } & \multicolumn{1}{c}{ Villa } \\
Evelyn & Isabel Riquelme \\
Ángela & Isabel Riquelme \\
Alejandra & Isabel Riquelme \\
Irene & Isabel Riquelme \\
Nancy & Isabel Riquelme \\
Silvia & Isabel Riquelme \\
Genesis & Isabel Riquelme \\
Vivian & Isabel Riquelme \\
Silvana & Isabel Riquelme \\
Irma & Isabel Riquelme \\
Julio & Isabel Riquelme \\
Mónica & Isabel Riquelme \\
Lorena & Isabel Riquelme \\
Ivonne & Isabel Riquelme \\
Elizabeth & Isabel Riquelme \\
Guillermina & Isabel Riquelme \\
Carmen & Isabel Riquelme \\
Roberto & Isabel Riquelme \\
Raquel & Isabel Riquelme \\
Ruth & Isabel Riquelme \\
Claudia & Isabel Riquelme \\
Rosa & Isabel Riquelme \\
Patricia & Isabel Riquelme \\
Sofia & Isabel Riquelme \\
Emily & Isabel Riquelme \\
Maciel & Isabel Riquelme \\
Rosana & Isabel Riquelme \\
Ana & Isabel Riquelme \\
María & Isabel Riquelme \\
Leonor & Isabel Riquelme \\
\hline & \\
\hline
\end{tabular}

Fuentes consultadas:

Ascher, Francoise (2004), Los nuevos principios del urbanismo, Alianza Editorial, Madrid.

Allen, Adriana (2003), "La interfase periurbana como escenario de cambio y acción hacia la sustentabilidad del desarrollo", CENDES, 20 (53), Universidad Central de Venezuela, Caracas, pp. 7-21.

Auge, Marc (1993), Los no lugares, espacios del anonimato, Editorial Gedisa, Barcelona. 
Brain Isabel y Francisco Sabatini (2006), "Relación entre el mercado del suelo y la política de vivienda social basada en el subsidio a la demanda: estudio en la Región Metropolitana de Santiago", Pro Urbana, Universidad de Chile, Santiago, pp. 2-13.

Brain, Isabel y Pía Mora (2016), "El barrio como motor del valor de la vivienda social en Chile: evidencia a partir de la Encuesta Panel de Vivienda 2010", Persona y Sociedad, 26 (1), Universidad Alberto Hurtado, Chile, pp. 113-145.

Cáceres, César (2015), "Ciudades satélites periurbanas en Santiago de chile: paradojas entre la satisfacción residencial y precariedad económica del periurbanita de clase media”, Revista INVI, 30 (85), Universidad de Chile, Santiago de Chile, pp. 83-108.

Caldeira, Teresa Pires (2000), City of walls. Crime, segregation and citizenship in Sau Paulo, University of California Press, Los Angeles.

Ducci, María Elena (1997), "Chile: el lado obscuro de una política de vivienda exitosa”, EURE, 23 (69), Pontificia Universidad Católica de Chile, Santiago de Chile, pp. 99-115.

Dureau, Francoise, Veronique Dupont, Éva Lelièvre, Jean Lévy y Lulle Thierry (2002), Metrópolis en movimiento: una comparación internacional, Alfaomega, Bogotá.

Ellen, Ingrid y Margery Turner (2001), "Does neighborhood matter? Assessing recent evidence", Housing policy debate, 8 (4), Taylor and Francis online, pp. 833-866.

Fishman, Robert (1987), Bourgeois utopias: the rise and fall of suburbia, Basic Books, New York.

Forrest, Ray (2008), "Who cares about neighbourhoods?", International Social Science Journal, 59 (191), Wiley Online Library, pp. 129141, doi: 10.1111/j.1468-2451.2009.00685.

Galster, George (2010), "The mechanism(s) of neighbourhood effects. Theory, evidence and policy implications", documento presentado en ESCR Seminar: Neighbourhood effects: Theory and evidence, 4-5 de febrero, 5t. Andrews University, Scotland. 
Galster, George y Sean Killen (1995), the geography of metropolitan opportunity: a reconnaissance and conceptual framework, Housing Policy Debate, 6 (1), Taylor and Francis online, pp. 7-43.

Garreau, Jean (1991), Edge City: life on the new frontier, Anchor Publisher, New York.

Graham, Stephen y Simon Marvin (2001), Splintering urbanism. Networked infrastructures, technological mobilities, and the urban condition, Routledge, London.

Google (2015), “Lampa”, <https://www.google.cl/maps/@-33.4856008,$70.7366967,58374 \mathrm{~m} /$ data=!3m1!1e3>, 22 de marzo del 2015.

Hanhörster, Heike (2001), "Whose neighbourhood is it? Ethnic diversity in urban spaces in Germany", GeoJournal, 51 (4), Kluwer Academic Publishers, Netherlands, pp. 329-338.

Heinrichs, Dirk, Henning Nuissl y Claudia Rodríguez (2009), "Dispersión urbana y nuevos desafíos para la gobernanza (metropolitana) en América Latina: el caso de Santiago de Chile", EURE, 35 (194), Pontificia Universidad Católica de Chile, Santiago de Chile, pp. 29-46.

Hidalgo, Rodrigo, Hugo Zunino y Lily Correa (2007), "El emplazamiento periférico de la vivienda social en el área metropolitana de Santiago de Chile: consecuencias socio espaciales y sugerencias para modificar los criterios actuales de localización", Scripta Nova: revista electrónica de geografía y ciencias sociales, XI (245), Universidad de Barcelona, Barcelona, pp. 741-798.

Iaquinta, David L, y Axel W. Drescher (2000), "Defining periurban: understanding rural-urban linkages and their connection to institutional contexts", documento presentado en el Tenth World Congress of the International Rural Sociology Association, 1 de agosto, Río de Janeiro.

Indovina, Francesco (2007), La ciudad de baja densidad. Lógicas, gestión y contención, Diputació Barcelona Xarxa de Municipis, Barcelona. 
Indovina, Francesco (1998), "Algunas consideracions sobre la ciutat difusa", Documents d'Análisi Geográfica, núm. 33, Universidad Autónoma de Barcelona, Barcelona, pp. 21-30.

Janoschka, Michael (2002), "El nuevo modelo de la ciudad latinoamericana: fragmentación y privatización”, EURE, 28 (85), Pontificia Universidad Católica de Chile, Santiago de Chile, pp. 11-20.

Kaztman, Ruben (2001), "Seducidos y abandonados: el aislamiento social de los pobres urbanos", Revista de la CEPAL, núm. 75, CEPAL, Santiago de Chile, pp. 171-189.

Kitchin, Rob y Nick Tate (2013), Conducting research in human geography: theory, methodology and practice, Routledge, London.

Knox, Paul y Steven Pinch (2014), Urban social geography: an introduction, Routledge, London.

La Tercera (2012), "Precios de vivienda en el Gran Santiago crecieron hasta 44\% en la última década", La Tercera, Grupo Copesa, Santiago de Chile, 23 de diciembre, p. 20.

Lefebvre, Henri (2013), La producción del espacio, Capitán Swing, Madrid.

Ley N 19859, Diario Oficial de la República de Chile, Santiago de Chile, 31 de enero de 2003.

Lupton, Ruth (2003), "Neighborhood effects can we measure them and does it matter?", case paper 73, Centre for Analysis of Social Exclusion, London.

Madanipour, Ali (2011), "Social exclusion and space", en R. LeGates y F. Stout, The city reader, Routledge, London, pp. 186-194.

Martinotti, Guido (1993), Metropoli, la nuova morfologia sociale della città, Il Mulino, Boloña.

Massey, Douglas y Nancy Denton (1988), “The dimensions of residential segregation”, Social forces, 67 (2), Oxford Academic, Oxford, pp. 281-315. 
McGregor, Duncan y David Simon (2012), "The peri-urban interface: approaches to sustainable natural and human resource use", Routledge, London.

MINVU (Ministerio de Vivienda y Urbanismo) (2015), "Propuesta de Políticas de Suelo para Integración Social”, Consejo Nacional de Desarrollo Urbano, Santiago de Chile.

Muñoz, Francesc (2008), Urbanalización. Paisajes comunes, lugares globales, Gustavo Gili, Barcelona.

Naranjo, Gloria (2008), "Instrumentos de ordenamiento territorial en el espacio rural: ¿Ordenan?”, en Gloria Yáńez, Arturo Orellana, Arturo Figueroa y Federico Arenas (eds.), Ciudad, poder, gobernanza, Instituto de Estudios Urbanos y Territoriales, Pontificia Universidad Católica de Chile, Santiago, pp. 175-200.

Rodríguez, Jorge y Camilo Arriagada (2004), "Segregación residencial en la ciudad latinoamericana”, EURE, 30 (89), Pontificia Universidad Católica de Chile, Santiago de Chile, pp. 5-24.

Sabatini, Francisco, Diego Campos, Gonzalo Quiero y Laura Blonda (2006), "Nuevas formas de pobreza y movilización popular en Santiago de Chile", en Gonzalo A. Saravi (ed.), De la pobreza a la exclusión: continuidades y rupturas de la cuestión social en América Latina, Prometeo Libros, Buenos Aires, pp. 97-136.

Salazar, Alejandro (2010), “Transformaciones socio-territoriales en la periferia metropolitana: la ciudad periurbana, estrategias locales y gobernanza en Santiago de Chile", Scripta Nova: revista electrónica de geografía y ciencias sociales, XIV (331), Universidad de Barcelona, Barcelona.

Sieverts, Thomas (2003), Cities without Cities. An interpretation of the Zwischenstadt, Spon Press, London.

Soja, Edward (2011), "Regional urbanization and the end of the metropolis era", en Gary Bridge y Sophie Watson, The new blackwell companion to the city, Wiley, Oxford, pp. 679-689.

Tacoli, Cecilia (1998), Rural-urban interactions: a guide to the literature, Environment and Urbanization, 10 (1), Sage, London, pp. 147-166. 
Tapia, Ricardo (2011), "Vivienda social en Santiago de Chile: análisis de su comportamiento locacional, periodo 1980-2002", Revista INVI, 26 (73), Universidad de Chile, Santiago de Chile, pp. 105-131.

Taschner, Suzana y Lucia Bogus (2001), "São Paulo, uma metrópole desigual”, EURE, 28 (80), Pontificia Universidad Católica de Chile, Santiago, pp. 87-120.

Tokman, Andrea (2006), "El Minvu, la politica habitacional y la expansión excesiva de Santiago", en Alexander Galetovic (ed.), Santiago: dónde estamos y hacia dónde vamos, Centro de Estudios Públicos, Santiago, pp. 489-520.

Trivelli, Pablo (2011), "La propuesta de modificación del Plan Regulador Metropolitano de Santiago PRMS 100 requiere una justificación más sólida”, EURE, 37 (111), Pontificia Universidad Católica de Chile, Santiago de Chile, pp. 179-184.

UN-Habitat (2009), "Planning sustainable cities", Global report on human settlements 2009, UN-Habitat, Nairobi.

Vidal, Tomeu y Enric Pol (2005), "La apropiación del espacio: una propuesta teórica para comprender la vinculación entre las personas y los lugares", Anuario de psicologia/The UB Journal of psychology, 36 (3), Universitat de Barcelona, Barcelona, pp. 281-298.

Recibido: 16 de junio 2015. Corregido: 9 de noviembre de 2015. Aceptado: 10 de febrero de 2016.

César Cáceres-Seguel. Chileno. Doctor y geógrafo. Actualmente es investigador en el Centro de Inclusión e Innovación Social de la Universidad Viña del Mar (Chile). Sus líneas de investigación son espacios residenciales e inequidad social; gobernabilidad urbano-metropolitana y desarrollo urbano y restructuración capitalista. Entre sus últimas publicaciones destacan: "Ciudades satélites periurbanas en Santiago de Chile: paradojas entre la satisfacción residencial y la precariedad económica del periurbanita de clase media”, Revista INVI, 30 (85), Universidad de Chile, Santiago de Chile, pp. 83-108 (2015); "Desarrollo residencial en Chile y la crisis del tercer lugar", Revista Planeo, núm. 19, Pontificia 
Universidad Católica de Chile, Santiago, <http://revistaplaneo.uc. cl/2015/01/13/desarrollo-residencial-en-chile-y-la-crisis-del-tercer-lugar/> (2015); "Ciudades satélites en Lampa, Santiago: un caso de cogobierno urbano entre el municipio y holdings inmobiliarios", Revista Cuadernos Geográficos, 55 (2), Universidad de Granada, Granada, (2016); "La urbanización de holdings empresariales en Chile 1990-2015: una industria de paisajes en serie", Revista $3 W$ de geografía y ciencias sociales, 21 (1.171), Universidad de Barcelona, Barcelona, pp.1-15 (2016); "Expansión residencial en comunas periurbanas de Santiago de Chile: organización territorial y nuevas lógicas de generación de centralidades urbanas en el Santiago periurbano", Revista de Diseño Urbano y Paisaje, núm. 30, Centro de Estudios Arquitectónicos y Urbanísticos del Paisaje, Santiago, pp. 18-24 (2015). 